REVISTA DE LA

UNIÓN MATEMÁTICA ARGENTINA

Vol. 59, No. 1, 2018, Pages 57-72

Published online: July 31, 2017

\title{
A NOTE ON WAVELET EXPANSIONS FOR DYADIC BMO FUNCTIONS IN SPACES OF HOMOGENEOUS TYPE
}

\author{
RAQUEL CRESCIMBENI AND LUIS NOWAK
}

Abstract. We give a characterization of dyadic BMO spaces in terms of Haar wavelet coefficients in spaces of homogeneous type.

\section{InTRODUCTION AND STATEMENT OF THE MAIN RESUlts}

The characterization of functional spaces via wavelet coefficients is one of the most studied properties in the general setting. In the Euclidean context there is, besides the well known Haar system, a great variety of wavelet systems with particular properties. Given the nature of the functional space, different systems of wavelets characterize such space $([12,13,17,18])$. For example, the Haar systems and others with regularity as the Daubechies's systems turn out to be unconditional bases for the classical Lebesgue spaces, and moreover there exist two positive and finite constants $c_{1}$ and $c_{2}$ such that

$$
c_{1}\|f\|_{L^{p}} \leq\left\|\left(\sum_{i \in \mathbb{Z}^{+}}\left|\left\langle f, \psi_{i}\right\rangle\right|^{2}\left|\psi_{i}\right|^{2}\right)^{1 / 2}\right\|_{L^{p}} \leq c_{2}\|f\|_{L^{p}},
$$

where $\left(\psi_{i}\right)_{i \in \mathbb{Z}^{+}}$is one of such systems of wavelets. Similar characterizations to those given in (1.1) hold for different systems of wavelets in other functional spaces, such as the Lorentz spaces $L^{p, q}$, the weighted Lebesgue spaces $L_{w}^{p}$ with $w$ in the Muckenhoupt class, and the Hardy spaces $H_{1}$, among others ([7, 12, 13, 17, 18]). In this note we consider the space of functions of dyadic bounded mean oscillation in spaces of homogeneous type. In the Euclidean case of the real line this space is defined by

$$
\mathrm{BMO}^{d}(\mathbb{R})=\left\{f \in L_{\mathrm{loc}}^{1}: \sup _{I} \frac{1}{|I|} \int_{I}\left|f(x)-f_{I}\right| d x<\infty\right\},
$$

where the supremum is taken over all the dyadic intervals $I$ of $\mathbb{R}$, with $f_{I}$ the $f$ average over $I$, and $|I|$ the Lebesgue measure of the interval $I$. For this functional space and the classical Haar system in $\mathbb{R}$ we have the following result [8].

2010 Mathematics Subject Classification. 42C15, 42B20, 28C15.

Key words and phrases. Dyadic BMO spaces, Carleson measure, Haar basis, spaces of homogeneous type. 
Proposition 1.1. The function $\phi$ belongs to $\mathrm{BMO}^{d}$ if and only if there exists a positive and finite constant $C$ such that

$$
\sum_{J \subseteq I, J \text { dyadic }}\left|c_{J}\right|^{2} \leq C|I|
$$

for all dyadic intervals $I$, where $c_{J}=\left\langle\phi, h_{J}\right\rangle=\int \phi(x) h_{J}(x) d x$ and for each dyadic interval $J$ the Haar function $h_{J}$ is defined as $\frac{1}{|J|^{1 / 2}}$ on the left half of $J$, as $\frac{-1}{|J|^{1 / 2}}$ on the right half of $J$, and zero otherwise.

The same result has been obtained in [13] and 17] for the non dyadic BMO and for wavelet systems with some regularity. In both works the regularity of the wavelets plays a central role. A generalization of this result in weighted BMO spaces can be found in [14]. In this paper we consider the problem for the dyadic case in the setting of spaces of homogeneous type. Our work is inspired in [2], where the authors give a characterization, via wavelets with regularity, of BMO spaces in the Euclidean context. This caracterization holds for the Meyer and Daubechies wavelets. However, in the general setting of spaces of homogeneous type one cannot expect any more smoothness than Lipschitz regularity. We prove an analogue of the results given in [2] for generalized dyadic $\mathrm{BMO}^{\mathcal{D}}$ spaces and Haar wavelets that we shall introduce later. Also, in our dyadic context in spaces of homogeneous type, new geometric ingredients appear underlying in the space and they are relevant in the proof of our results. Notice that since the Haar wavelets are not continuous, for our proof we use only the properties of the dyadic family that supports the Haar systems and the regularity arguments in [2] are replaced by that geometric argument.

In section 2 we shall introduce the dyadic families $\mathcal{D}$ and the Haar systems $\mathcal{H}$ in spaces of homogeneous type. Then in section 3 we shall define the spaces we will work with along the paper: the dyadic spaces $\mathrm{BMO}^{\mathcal{D}}, H_{1}^{\mathcal{D}}$, and the Carleson class $\mathcal{C}$ in spaces of homogeneous type, with the respective norms $\|\cdot\|_{\mathrm{BMO}^{\mathcal{D}}},\|\cdot\|_{H^{\mathcal{D}}}$ and $\|\cdot\|_{\mathcal{C}}$. The main results of this note are the following three theorems that we shall prove in sections 4 and 5 .

Theorem 1.2. Let $\mathcal{H}$ be a Haar system associated with a dyadic family $\mathcal{D}$. Let $f \in \mathrm{BMO}^{\mathcal{D}}$. Then the sequence $\mathfrak{C}=\{\langle f, h\rangle\}_{h \in \mathcal{H}}$ belongs to the Carleson class $\mathcal{C}$. Moreover, there exists a positive constant $C$ such that

$$
\|\mathfrak{C}\|_{\mathcal{C}} \leq C\|f\|_{\mathrm{BMO}^{\mathcal{D}}}
$$

Theorem 1.3. Let $\mathcal{H}$ be a Haar system associated with a dyadic family $\mathcal{D}$. If $\mathfrak{C}=\left\{c_{h}\right\}_{h \in \mathcal{H}}$ is a sequence of real numbers vanishing except for a finite subset of $\mathcal{H}$ then the function $f=\sum_{h \in \mathcal{H}} c_{h} h$ belongs to $\mathrm{BMO}^{\mathcal{D}}$. Moreover, there exists a positive constant $C$ such that

$$
\|f\|_{\mathrm{BMO}^{\mathcal{D}}} \leq C\|\mathfrak{C}\|_{\mathcal{C}}
$$

Theorem 1.4. Let $\mathcal{H}$ be a Haar system associated with a dyadic family $\mathcal{D}$. Let $\mathfrak{C}=\left\{c_{h}\right\}_{h \in \mathcal{H}}$ be a sequence of real numbers that belong to the Carleson class $\mathcal{C}$. 
Then, the series

$$
\sum_{h \in \tilde{\mathcal{H}}} c_{h} h
$$

converges in the sense of the weak-* topology of $\mathrm{BMO}^{\mathcal{D}}$ to a function $f \in \mathrm{BMO}^{\mathcal{D}}$.

\section{Dyadic families And HaAR systems in spaces of homogeneOUS TYPe}

We first briefly recall the basic properties of the general theory of spaces of homogeneous type. Assume that $X$ is a set; a nonnegative symmetric function $d$ on $X \times X$ is called a quasi-distance if there exists a constant $K$ such that

$$
d(x, y) \leq K[d(x, z)+d(z, y)],
$$

for every $x, y, z \in X$, and $d(x, y)=0$ if and only if $x=y$.

We shall say that $(X, d, \mu)$ is a space of homogeneous type if $d$ is a quasi-distance on $X, \mu$ is a positive Borel measure defined on a $\sigma$-algebra of subsets of $X$ which contains the balls, and there exists a constant $A$ such that

$$
0<\mu(B(x, 2 r)) \leq A \mu(B(x, r))<\infty
$$

holds for every $x \in X$ and every $r>0$.

In [16] the authors prove that each quasi-metric space is metrizable and that $d$ is equivalent to $\rho^{\beta}$, where $\rho$ is a distance on $X$ and $\beta \geq 1$. Therefore we shall assume throughout this paper that $d$ is actually a distance on $X$, in other words that $K=1$ in 2.1 .

The construction of dyadic type families of subsets in metric or quasi-metric spaces with some inner and outer metric control of the sizes of the dyadic sets is given in [9]. These families satisfy all the relevant properties of the usual dyadic cubes in $\mathbb{R}^{n}$ and are the basic tool to build wavelets on a metric space of homogeneous type (see [1] or [3]). The notion of dyadic families that we will consider here is contained in the following definition (see [6]).

Definition 2.1 (The class $\mathfrak{D}(\delta)$ of all dyadic families). Let $(X, d, \mu)$ be a metric space of homogeneous type. We say that $\mathcal{D}=\bigcup_{j \in \mathbb{Z}} \mathcal{D}^{j}$ is a dyadic family on $X$ with parameter $\delta \in(0,1)$-briefly, that $\mathcal{D}$ belongs to $\mathfrak{D}(\delta)$ - if each $\mathcal{D}^{j}$ is a family of Borel subsets $Q$ of $X$ such that

(d.1) for every $j \in \mathbb{Z}$ the cubes in $\mathcal{D}^{j}$ are pairwise disjoint;

(d.2) for every $j \in \mathbb{Z}$ the family $\mathcal{D}^{j}$ almost covers $X$ in the sense that $\mu(X \backslash$ $\left.\bigcup_{Q \in \mathcal{D}^{j}} Q\right)=0$;

(d.3) if $Q \in \mathcal{D}^{j}$ and $i<j$, then there exists a unique $\tilde{Q} \in \mathcal{D}^{i}$ such that $Q \subseteq \tilde{Q}$;

(d.4) if $Q \in \mathcal{D}^{j}$ and $\tilde{Q} \in \mathcal{D}^{i}$ with $i \leq j$, then either $Q \subseteq \tilde{Q}$ or $Q \cap \tilde{Q}=\emptyset$;

(d.5) there exist two constants $a_{1}$ and $a_{2}$ such that for each $Q \in \mathcal{D}^{j}$ there exists a point $x \in Q$ that satisfies $B\left(x, a_{1} \delta^{j}\right) \subseteq Q \subseteq B\left(x, a_{2} \delta^{j}\right)$.

The following properties can be deduced from (d.1)-(d.5):

(d.6) there exists a positive integer $N$ depending on $a_{i}, i=1,2$ in (d.5) and on the doubling constant $A$ such that for every $j \in \mathbb{Z}$ and all $Q \in \mathcal{D}^{j}$ the 
inequalities $1 \leq \#(\mathcal{L}(Q)) \leq N$ hold, where $\mathcal{L}(Q)=\left\{Q^{\prime} \in \mathcal{D}^{j+1}: Q^{\prime} \subseteq Q\right\}$ and $\#(B)$ denotes the cardinal of $B$;

(d.7) there exists a positive constant $c$ such that $\mu(Q) \leq c \mu\left(Q^{\prime}\right)$ for all $Q \in \tilde{\mathcal{D}}$ and every $Q^{\prime} \in \mathcal{L}(Q)$.

It is easy to give examples of dyadic systems $\mathcal{D}$ such that a dyadic cube $Q$ belongs to different levels $j \in \mathbb{Z}$. In fact, the usual dyadic cube in the real line intersected with the natural set $\mathbb{N}$ is a dyadic family in the space of homogeneous type $(\mathbb{N}, d, \mu)$ with $d$ the usual metric and $\mu$ the counting measure. In this case, the points turn out to be dyadic cubes belonging to different and infinite resolution levels of the space's dyadic decomposition. We are interested in the identification of those scales and places of partition which shall give rise to the Haar functions. This induces the definition of a subfamily of $\mathcal{D}$ containing all dyadic cubes in $\mathcal{D}$ with non-trivial offspring.

Definition 2.2 (The subfamily $\tilde{\mathcal{D}}$ of a $\mathcal{D}$ in $\mathfrak{D}(\delta)$ ). For each $\mathcal{D}$ in $\mathfrak{D}(\delta)$ and for each $j \in \mathbb{Z}$ we consider the families

$$
\tilde{\mathcal{D}}^{j}=\left\{Q \in \mathcal{D}^{j}: \#\left(\left\{Q^{\prime} \in \mathcal{D}^{j+1}: Q^{\prime} \subseteq Q\right\}\right)>1\right\} .
$$

We define

$$
\tilde{\mathcal{D}}=\bigcup_{j \in \mathbb{Z}} \tilde{\mathcal{D}}^{j}
$$

Properties (d.1)-(d.6) allow us to obtain the following result.

Proposition 2.3. Let $(X, d, \mu)$ be a metric space of homogeneous type and let $\mathcal{D}$ be in $\mathfrak{D}(\delta)$. Then

(a) The families $\tilde{\mathcal{D}}^{j}, j \in \mathbb{Z}$, are pairwise disjoint.

(b) The function $\mathcal{J}: \tilde{\mathcal{D}} \longrightarrow \mathbb{Z}$ given by $Q \mapsto \mathcal{J}(Q)$ if $Q \in \tilde{\mathcal{D}}^{\mathcal{J}(Q)}$ is well defined.

We would like to note that the point $x \in Q$ given in (d.5) cannot be unique. It will be important in the sequel to identify one of them. This induces the following definition.

Definition 2.4. Let $(X, d, \mu)$ be a space of homogeneous type and let $\mathcal{D}$ be a dyadic family in $\mathfrak{D}(\delta)$. For each $j \in \mathbb{Z}$ we define a function $\mathcal{P}^{j}$, that we will call point central function of level $j$, as $\mathcal{P}^{j}: \mathcal{D}^{j} \longrightarrow X$ given by $\mathcal{P}^{j}(Q)=x_{Q} \in Q$ such that $a_{1} \delta^{j} \leq d\left(x_{Q}, X \backslash Q\right)$.

From the condition (d.5) we get that for each integer $j$ the point central function of level $j, \mathcal{P}^{j}$, is well defined. The next result contains the main property of this function. We recall that a set $A$ in a metric space $(X, d)$ is $\alpha$-dispersed if $d(x, y) \geq \alpha$ for all $x, y \in A$.

Proposition 2.5. Let $(X, d, \mu)$ be a space of homogeneous type, let $\mathcal{D}$ be a dyadic family in $\mathfrak{D}(\delta)$ and let $\left\{\mathcal{P}^{j}: j \in \mathbb{Z}\right\}$ be a family of point central functions. Then

(1) the set $\mathcal{P}^{j}\left(\mathcal{D}^{j}\right)$ is $\frac{a_{1}}{2} \delta^{j}$-dispersed for each $j \in \mathbb{Z}$;

(2) the set $\mathcal{P}=\bigcup_{j \in \mathbb{Z}} \mathcal{P}^{j}\left(\mathcal{D}^{j}\right)$ is dense in $X$. 
Proof. First we prove (1). Let $j \in \mathbb{Z}$ and let $x, y$ be two points in $\mathcal{P}^{j}\left(\mathcal{D}^{j}\right)$, with $x \neq y$. Let $Q_{1}=\left(\mathcal{P}^{j}\right)^{-1}(x)$ and $Q_{2}=\left(\mathcal{P}^{j}\right)^{-1}(y)$. Notice that $Q_{1}$ and $Q_{2}$ belong to $\mathcal{D}^{j}$ and that $\mathcal{P}_{j}$ is one to one. Thus, from (d.1), $Q_{1} \cap Q_{2}=\emptyset$. Suppose that $d(x, y)<\frac{a_{1}}{2} \delta^{j}$. Then $y \in B\left(x, \frac{a_{1}}{2} \delta^{j}\right)$ and hence $y \in Q_{1}$, which is a contradiction. Therefore $d(x, y) \geq \frac{a_{1}}{2} \delta^{j}$ and $\mathcal{P}^{j}\left(\mathcal{D}^{j}\right)$ is $\frac{a_{1}}{2} \delta^{j}$-dispersed.

For proving (2), we take $x \in X$ and $\varepsilon>0$. We fix $j \in \mathbb{Z}$ such that $2 a_{2} \delta^{j}<\varepsilon / 2$. We first suppose that $x \in \bigcup_{Q \in \mathcal{D}^{j}} Q$ and take $Q$, the unique dyadic cube in $\mathcal{D}^{j}$ such that $x \in Q$. Then, from (d.5), we get that $x \in B\left(\mathcal{P}^{j}(Q), 2 a_{2} \delta^{j}\right)$ and hence, from the selection of the integer $j$, we have that $d\left(x, \mathcal{P}^{j}(Q)\right)<\varepsilon$. On the other hand, if $x \in X \backslash \bigcup_{Q \in \mathcal{D}^{j}} Q$ then $x \in \partial(Q)$ for some $Q$ in $\mathcal{D}^{j}$. Since the balls have positive measure, by (d.2) we have that $B_{d}(x, \varepsilon / 2) \cap Q^{\prime} \neq \emptyset$ for some $Q^{\prime} \in \mathcal{D}^{j}$ such that $\bar{Q} \cap \overline{Q^{\prime}} \neq \emptyset$. That is, there exists $y \in Q^{\prime}$ such that $d(x, y)<\varepsilon / 2$. Thus, from (d.5) and the selection of $j$ we obtain that $d\left(x, \mathcal{P}^{j}\left(Q^{\prime}\right)\right) \leq d(x, y)+d\left(y, \mathcal{P}^{j}\left(Q^{\prime}\right)\right) \leq$ $\varepsilon / 2+\varepsilon / 2$.

In the sequel we shall need the following geometric characterization of atoms in spaces of homogeneous type via the subfamily $\tilde{\mathcal{D}}$. We recall that a point $x \in X$, where $(X, d, \mu)$ is a space of homogeneous type, is an atom in $X$ if $\mu(\{x\})>0$. A well know result of Macías and Segovia ([16]) gives a characterization of atoms through the metric in $(X, d, \mu)$. More precisely, the authors prove that the point $x$ is an atom in $X$ if and only if there exists a positive and finite constant $r$ such that $B(x, r) \cap X=\{x\}$. We shall use this characterization to prove that the subfamily of non trivial offspring $\tilde{\mathcal{D}}$ of the dyadic family $\mathcal{D}$ identifies atoms. In fact, we have the following result.

Proposition 2.6. Let $(X, d, \mu)$ be a space of homogeneous type and let $\mathcal{D}$ be a dyadic family in $\mathfrak{D}(\delta)$. Let $\tilde{\mathcal{D}}$ be the subfamily of non trivial offspring of $\mathcal{D}$. Then

$$
\mathcal{D}=\tilde{\mathcal{D}} \cup\{\{x\}: \mu(x)>0\},
$$

where the union is disjoint.

Proof. We shall prove first that $\mathcal{D} \subseteq \tilde{\mathcal{D}} \cup\{\{x\}: \mu(x)>0\}$. Notice that we only need to consider the case where $Q$ does not belong to $\tilde{\mathcal{D}}$. Let $j$ be an integer such that $Q \in \mathcal{D}^{j}$. From the definition of $\tilde{\mathcal{D}}$ and since $Q \notin \tilde{\mathcal{D}}$ we have that $Q \in \mathcal{D}^{j+n}$ for every natural number $n$. Then, for all positive integers $n$ and each dyadic cube $Q^{\prime} \in \mathcal{D}^{j+n}$ with $Q^{\prime} \subseteq Q$ we get that $Q^{\prime}=Q$. Now, we fix for every positive integer $n$ the following function $\mathcal{P}^{j+n}: \mathcal{D}^{j+n} \longrightarrow X$ with $\mathcal{P}^{j+n}(Q)=x$ for some $x \in Q$. We write $x_{n}=\mathcal{P}^{j+n}(Q)$ and consider the ball $B_{n}=B_{d}\left(x_{n}, 2 a_{2} \delta^{j+n}\right)$, where $a_{2}$ is the constant in (d.5) for the cube $Q$. Thus we get that $Q \subseteq B_{n}$ for each $n$. Then since the radii of the balls $B_{n}$ go to zero, by a standard argument we have that $x_{n}=x_{n+1}$ and $Q=\left\{x_{n}\right\}$. From the result of Macías and Segovia we have that $x_{n}$ is an atom in $X$.

Now we prove that $\tilde{\mathcal{D}} \cup\{\{x\}: \mu(x)>0\} \subseteq \mathcal{D}$. Notice that we only need to prove that if $x$ is an atom then $\{x\} \in \mathcal{D}$. We recall that from $(\mathrm{d} .2), \mu\left(\bigcup_{Q \in \mathcal{D}}(\partial Q)\right)=0$; then for each integer $j$ there exists a dyadic cube $Q_{j} \in \mathcal{D}^{j}$ such that $x \in Q^{j}$. For each integer $j$ we fix a point central function $\mathcal{P}^{j}: \mathcal{D}^{j} \longrightarrow X$. From Macías and 
Segovia we get that $x$ is an isolated point and therefore there exists an $\varepsilon>0$ such that

$$
B_{d}(x, \varepsilon) \cap X=\{x\} .
$$

On the other hand, from Proposition 2.5item (2), we have that the set $\bigcup_{j \in \mathbb{Z}} \mathcal{P}^{j}\left(\mathcal{D}^{j}\right)$ is dense in $X$. Thus we can take $j_{0} \in \mathbb{Z}$ and $Q \in \mathcal{D}^{j_{0}}$ such that $d\left(x, \mathcal{P}^{j_{0}}(Q)\right)<\varepsilon / 2$ and $2 a_{2} \delta^{j_{0}}<\varepsilon / 2$, where $a_{2}$ is the constant in (d.5) for the cube $Q$. Therefore $Q=Q_{j_{0}}=\{x\}$.

Having introduced the dyadic cubes, we now define the other basic objects with which we will work along the present article: the systems of Haar type associated with a dyadic family (see [6]).

Definition 2.7. Let $\mathcal{D}$ be a dyadic family on $(X, d, \mu)$ such that $\mathcal{D} \in \mathfrak{D}(\delta)$. A system $\mathcal{H}$ of simple Borel measurable real functions $h$ on $X$ is a Haar system associated with $\mathcal{D}$ if it satisfies:

(h.1) For each $h \in \mathcal{H}$ there exists a unique $j \in \mathbb{Z}$ and a cube $Q=Q_{h} \in \tilde{\mathcal{D}}^{j}$ such that $\{x \in X: h(x) \neq 0\} \subseteq Q$, and this property does not hold for any cube in $\mathcal{D}^{j+1}$.

(h.2) For every $Q \in \tilde{\mathcal{D}}$ there exist exactly $M_{Q}=\#(\mathcal{L}(Q))-1 \geq 1$ functions $h \in \mathcal{H}$ such that (h.1) holds. We shall write $\mathcal{H}_{Q}$ to denote the set of all these functions $h$.

(h.3) For each $h \in \mathcal{H}$ we have that $\int_{X} h d \mu=0$.

(h.4) For each $Q \in \tilde{\mathcal{D}}$ let $V_{Q}$ denote the vector space of all functions on $Q$ which are constant on each $Q^{\prime} \in \mathcal{L}(Q)$. Then the system $\left\{\frac{\chi_{Q}}{(\mu(Q))^{1 / 2}}\right\} \cup \mathcal{H}_{Q}$ is an orthonormal basis for $V_{Q}$.

It is easy to show, following the proof in [1] (see also [3]), that given $\mathcal{D}$ in $\mathfrak{D}(\delta)$ it is always possible to construct Haar systems supported on the elements $Q$ of $\tilde{\mathcal{D}}$. This means that there exist systems $\mathcal{H}$ of functions $h$ on $X$ satisfying (h.1) to (h.4) for all $\mathcal{D}$ in $\mathfrak{D}(\delta)$. Observe also that from (d.7) we get that there exists a positive constant $C$ such that

$$
\|h\|_{\infty} \leq C \mu\left(Q_{h}\right)^{-1 / 2},
$$

for all $h \in \mathcal{H}$. Here, as usual, $\|f\|_{\infty}$ is the $L^{\infty}$-norm of the function $f$ which is defined as the $\mu$-essential least upper bound of $f$. Also, the Haar system $\mathcal{H}$ is an orthonormal basis of $L^{2}(X, \mu)$.

\section{The Dyadic SPACEs BMO ${ }^{\mathcal{D}}$ And $H_{1}^{\mathcal{D}}$ AND SEQUential SPACES of CARLESON TYPE}

In this section we define three spaces that we will relate later via Haar wavelets: the Carleson class, the dyadic BMO space, and the dyadic Hardy spaces.

We first introduce a Carleson type condition generalizing to our dyadic context in spaces of homogeneous type those given by Lemarié and Meyer in [15] and Aimar and Bernardis in [2]. We shall say that the sequence $\mathfrak{C}=\left\{c_{h}\right\}_{h \in \mathcal{H}}$ over the Haar system $\mathcal{H}$ belongs to the sequential space of Carleson type $\mathcal{C}$, or simply that it 
belongs to the Carleson class $\mathcal{C}$, if there exists a positive constant $A$ such that the inequality

$$
\sum_{h \in \mathcal{H}: \operatorname{supp}(h) \subseteq R}\left|c_{h}\right|^{2} \leq A \mu(R)
$$

holds for every dyadic cube $R \in \mathcal{D}$. We will denote with $\|\mathfrak{C}\|_{\mathcal{C}}$ the infimum of the squares of those constants $A$, so that

$$
\mathcal{C}=\left\{\mathfrak{C}=\left\{c_{h}\right\}_{h \in \mathcal{H}}:\|\mathfrak{C}\|_{\mathcal{C}}<\infty\right\} .
$$

Notice that our definition is apparently different from that given in [2] because their sum is over dyadic cubes. But in the real line for each dyadic interval $I$ there exists a unique wavelet function supported in $I$. In [15] the authors consider the $\mathbb{R}^{n}$ case; in such a context for each cube they have a uniform number of wavelets supported on it. Our general context is a little different. In fact, we do not have a uniform number of wavelets supported in each dyadic cube $Q$; however by (d.6) this number is uniformly bounded. If we consider as spaces of homogeneous type the $\mathbb{R}^{n}$ spaces, our definition is the same as that given in [15].

For introducing dyadic $p$-bounded mean oscillation functions adequate in our setting of measure metric spaces, it will be necessary the following definition that generalizes to our dyadic families in $\mathfrak{D}(\delta)$ the notion of quadrant given in [3] for Christ's dyadic cubes, where the authors give a detailed presentation.

Definition 3.1. Let $\mathcal{D}$ be a dyadic family in the class $\mathfrak{D}(\delta)$. We define, for each dyadic cube $Q$ in $\mathcal{D}$, the quadrant of $X$ that contains the cube $Q, \mathbf{C}(Q)$, by

$$
\mathbf{C}(Q)=\bigcup_{\left\{Q^{\prime} \in \mathcal{D}: Q \subseteq Q^{\prime}\right\}} Q^{\prime} .
$$

Following the lines in [3] for the case of Christ's dyadic cube, from (d.6) and since all the dyadic cubes $Q$ in $\mathcal{D}$ are spaces of homogeneous type with doubling uniform constant, we can prove the next result that will be important in section 5 .

Proposition 3.2. Let $(X, d, \mu)$ be a space of homogeneous type and let $\mathcal{D}$ be a dyadic family in the class $\mathfrak{D}(\delta)$. Then there exists a positive integer $N$ (that depends on the geometric constants of $(X, d, \mu)$ ) and disjoint dyadic cubes $Q_{\alpha}, \alpha=1, \ldots, N$, such that

$$
X \backslash \partial \bigcup_{Q \in \mathcal{D}} Q=\bigcup_{\alpha=1, \ldots, N} \mathbf{C}_{\alpha},
$$

where $\mathbf{C}_{\alpha}=\mathbf{C}\left(Q_{\alpha}\right)$ and $\partial A$ denotes the border of the set $A$. That is, there exists a finite number of quadrants that are a partition of $X$.

With the above notation we shall write $\mathbf{C}(X, \mathcal{D})$ to denote the family of quadrants $\left\{\mathbf{C}_{\alpha}: \alpha=1, \ldots, N\right\}$ associated with a dyadic family $\mathcal{D}$ given by Proposition 3.2

The string of spaces $\mathrm{BMO}_{p}^{\mathcal{D}}$ of all functions of $p$-bounded mean oscillation, $1 \leq$ $p<\infty$, is defined by $\mathrm{BMO}_{p}^{\mathcal{D}}=\left\{f:\|f\|_{*, p}<\infty\right\}$, where

$$
\|f\|_{*, p}=\sup _{Q \in \mathcal{D}}\left(\frac{1}{\mu(Q)} \int_{Q}\left|f(x)-f_{Q}\right|^{p} d \mu(x)\right)^{1 / p}
$$


and $f_{Q}=\frac{1}{\mu(Q)} \int_{Q} f d \mu$. In the case $p=1$ we shall write $\mathrm{BMO}^{\mathcal{D}}$ instead of $\mathrm{BMO}_{1}^{\mathcal{D}}$. Notice that our dyadic setting is reflected in the fact that the functions in $\mathrm{BMO}_{p}^{\mathcal{D}}$ are equivalence classes: $f$ is equivalent to $g$ if and only if $f-g$ is constant over each quadrant of $X$. Thus, from Proposition 3.2 we have that there exist two positive and finite constants $c_{1}$ and $c_{2}$ such that

$$
c_{1}\|f\|_{*, p} \leq \sum_{\alpha=1, \ldots, N}\left\|f_{\alpha}\right\|_{*, p} \leq c_{2}\|f\|_{*, p},
$$

where the function $f_{\alpha}$ is $f$ on each $\mathbf{C}_{\alpha} \in \mathbf{C}(X, \mathcal{D})$ and zero otherwise.

On the other hand, our definition of a dyadic family allows us to have a CalderónZygmund type decomposition associated with such a family. Moreover, considering each cube as a space of homogeneous type, such a decomposition can be done in each dyadic cube. In such case the doubling constant is uniform over all dyadic cubes and therefore following the proof of Theorem 6.16 in 18 we obtain the following dyadic version of the John-Nirenberg inequality.

Theorem 3.3. Let $\mathcal{D}$ be a dyadic family in the class $\mathfrak{D}(\delta)$. Then there exist two positive constants $C_{1}$ and $C_{2}$ such that for every function $f \in \mathrm{BMO}_{1}^{\mathcal{D}}$, every dyadic cube $Q \in \mathcal{D}$ and every $t \geq 0$ we have the following inequality:

$$
\mu\left(\left\{x \in Q:\left|f(x)-f_{Q}\right|>t\right\}\right) \leq C_{1} \mu(Q) e^{-\frac{C_{2} t}{\|f\|_{*, 1}}} .
$$

One of the main consequences of this important result is the equivalence between the norms of all dyadic $p$-bounded mean oscillation spaces.

Corollary 3.4. Let $\mathcal{D}$ be a dyadic family in the class $\mathfrak{D}(\delta)$ and $1 \leq p<\infty$. Then there exists a positive constant $C$ such that for each function $f \in \mathrm{BMO}_{p}^{\mathcal{D}}$ we have

$$
\|f\|_{*, 1} \leq\|f\|_{*, p} \leq C\|f\|_{*, 1} .
$$

Any of the equivalent norms $\|\cdot\|_{*, p}$ will be denoted by $\|\cdot\|_{\mathrm{BMO}^{\mathcal{D}}}$.

We introduce, as in [5], the dyadic Hardy space $H_{1}^{\mathcal{D}}$ on a space of homogeneous type. First of all we give the definition of dyadic atom associated with a dyadic family $\mathcal{D}$ in the class $\mathfrak{D}(\delta)$.

Definition 3.5. Let $\mathcal{D}$ be a dyadic family in the class $\mathfrak{D}(\delta)$. For $1<q \leq \infty$ we shall say that a function $a$ defined on $X$ is a dyadic $q$-atom associated with $\mathcal{D}$ -briefly, that $a \in \mathcal{A}_{q, \mathcal{D}}$ - if there exists a dyadic cube $Q$ in $\mathcal{D}$ such that

(a1) $\operatorname{supp}(a(x)) \subseteq Q$.

(a2) $\int_{X} a(x) d \mu(x)=0$.

(a3) $\|a\|_{L^{q}(X, \mu)} \leq(\mu(Q))^{\frac{1}{q}-1}$ if $q<\infty$, and $\left.\|a\|_{L^{\infty}(X, \mu)} \leq \mu(Q)\right)^{-1}$ if $q=\infty$.

For $1<q \leq \infty$, the spaces $H_{1}^{q, \mathcal{D}}$ are defined as follows.

Definition 3.6. Let $\mathcal{D}$ be a dyadic family in the class $\mathfrak{D}(\delta)$. For $1<q \leq \infty$ we define the space $H_{1}^{q, \mathcal{D}}$ as the linear space of all functions $f$ on $X$-identifying those that are equal almost everywhere with respect to $\mu$ - that can be written as

$$
f=\sum_{n \in \mathbb{Z}^{+}} \lambda_{n} a_{n}, \quad \text { with } \sum_{n \in \mathbb{Z}^{+}}\left|\lambda_{n}\right|<\infty
$$


where $a_{n} \in \mathcal{A}_{q, \mathcal{D}}$ for each $n$ and the convergence is in the $L^{1}(X, \mu)$ norm.

For each function $f$ in $H_{1}^{q, \mathcal{D}}$ we define the number

$$
\|f\|_{1, q, \mathcal{D}}=\inf \left\{\sum_{n \in \mathbb{Z}^{+}}\left|\lambda_{n}\right|<\infty: f=\sum_{n \in \mathbb{Z}^{+}} \lambda_{n} a_{n}, a_{n} \in \mathcal{A}_{q, \mathcal{D}}\right\} .
$$

The following result is a consequence of Definition 3.5

Proposition 3.7. Let $\mathcal{D}$ be a dyadic family in the class $\mathfrak{D}(\delta)$.

(1) If $1<q_{1}<q_{2} \leq \infty$, then $\mathcal{A}_{q_{2}, \mathcal{D}} \subseteq \mathcal{A}_{q_{1}, \mathcal{D}}$. Moreover, if $1<q \leq \infty$ and $a=a(x)$ is a q-dyadic atom, then $\|a\|_{L^{1}(X, \mu)} \leq 1$.

(2) For each $1<q_{1}<q_{2} \leq \infty$ we have that $H_{1}^{q_{2}, \mathcal{D}}(X, d, \mu) \subseteq H_{1}^{q_{1}, \mathcal{D}}(X, d, \mu)$ and $\|f\|_{L^{1}(X, \mu)} \leq\|f\|_{1, q_{1}, \mathcal{D}} \leq\|f\|_{1, q_{2}, \mathcal{D}}$.

(3) $\mid\|\cdot\|_{1, q, \mathcal{D}}$ is a norm and $\left(H_{1}^{q, \mathcal{D}},\|\cdot\| \|_{1, q, \mathcal{D}}\right)$ is a Banach space, for each $1<$ $q \leq \infty$.

(4) If $f \in H_{1}^{q, \mathcal{D}}$ then $\int_{X} f(x) d \mu(x)=0$.

(5) If $Q$ is a dyadic cube in $\mathcal{D}$ such that $f \in L^{q}(Q, \mu), f(x)=0$ for each $x$ in $X \backslash Q$ and $\int_{Q} f(x) d \mu(x)=0$, then $f \in H_{1}^{q, \mathcal{D}}$.

One of the tools that we will use for the proof of our main results is the following duality result for the dyadic spaces $H_{1}^{\mathcal{D}}$ and $\mathrm{BMO}^{\mathcal{D}}$ in spaces of homogeneous type. As a consequence of this duality and by a classical functional analysis result we obtain that $\mathrm{BMO}^{\mathcal{D}}$ is a Banach space.

Theorem 3.8. Let $\mathcal{D}$ be a dyadic family in the class $\mathfrak{D}(\delta)$. For $1<q \leq \infty$ the spaces $H_{1}^{q, \mathcal{D}}$ coincide and the norms $\|\cdot\| \|_{1, q, \mathcal{D}}$ are equivalent. This unique space will be denoted by $H_{1}^{\mathcal{D}}$ and any of the norms $\|\cdot\| \|_{1, q, \mathcal{D}}$ will be denoted by $\|\cdot\| \|_{1, \mathcal{D}}$. We have also that $\left(H_{1}^{\mathcal{D}}\right)^{*}$, the dual of $H_{1}^{\mathcal{D}}$, is $\mathrm{BMO}^{\mathcal{D}}$ in the sense that for each continuous linear functional $\varphi$ on $H_{1}^{\mathcal{D}}$ there exists a unique (up to functions which are constant on each quadrant) function $b \in \mathrm{BMO}^{\mathcal{D}}$ such that if $f$ is any finite sum of atoms we have that $\varphi(f)=\int_{X} b f d \mu$ and that the $\mathrm{BMO}^{\mathcal{D}}$ norm of $b$ and the functional norm of $\varphi$ are equivalent.

As far as we know the proof of this result has not been detailed in other works. Moreover, it is not a consequence of the Coifman-Weiss duality result in spaces of homogeneous type. However, following [18] adapted to this setting it is possible to prove it.

\section{Proof of Theorem 1.2 and Theorem 1.3}

Proof of Theorem 1.2. Let be $R$ a dyadic cube in $\mathcal{D}$ and let $f$ be a function in $\mathrm{BMO}^{\mathcal{D}}$. Then we rewrite the function $f$ in the following way: $f=f_{1}+f_{2}+f_{R}$, where $f_{R}=\frac{1}{\mu(R)} \int_{R} f d \mu, f_{1}=\left(f-f_{R}\right) \chi_{R}$ and $f_{2}=\left(f-f_{R}\right) \chi_{X \backslash R}$. So, from (h.3) we have that $\left\langle h, f_{R}\right\rangle=0$ for every Haar function $h \in \mathcal{H}$. On the other hand, since $\operatorname{supp}(h) \cap \operatorname{supp}\left(f_{2}\right)=\emptyset$ for every Haar function $h \in \mathcal{H}$ with $\operatorname{supp}(h) \subseteq R$, we have also that $\left\langle h, f_{2}\right\rangle=0$ for all such functions $h$. 
So, we get that

$$
\left(\sum_{h \in \mathcal{H}: \operatorname{supp}(h) \subseteq R}|\langle f, h\rangle|^{2}\right)^{\frac{1}{2}}=\left(\sum_{h \in \mathcal{H}: \operatorname{supp}(h) \subseteq R}\left|\left\langle f_{1}, h\right\rangle\right|^{2}\right)^{\frac{1}{2}} .
$$

Then, from Parseval's inequality and the fact that $f$ belongs to $\mathrm{BMO}^{\mathcal{D}}$ considering the norm in $\mathrm{BMO}_{2}^{\mathcal{D}}$, we obtain that

$$
\begin{aligned}
\left(\sum_{h \in \mathcal{H}: \operatorname{supp}(h) \subseteq R}|\langle f, h\rangle|^{2}\right)^{\frac{1}{2}} & \leq\left\|f_{1}\right\|_{2} \\
& =\left(\int_{R}\left|f-f_{R}\right|^{2} d \mu\right)^{\frac{1}{2}} \\
& \leq C\|f\|_{\mathrm{BMO}^{\mathcal{D}}} .
\end{aligned}
$$

Proof of Theorem 1.3. We first notice that, since every Haar function $h \in \mathcal{H}$ belongs to $L^{\infty}(X, \mu)$, then $h$ belongs to $\mathrm{BMO}^{\mathcal{D}}$ for all $h \in \mathcal{H}$. So, since $f$ is a finite sum of multiples of Haar functions, we have that $f \in \mathrm{BMO}^{\mathcal{D}}$.

Now, to prove the inequality in norm, we fix a dyadic cube $R \in \mathcal{D}$ and a point $x_{0} \in R$. Notice that if $R \in \mathcal{D} \backslash \tilde{\mathcal{D}}$ by Proposition 2.6 we get that $R=\left\{x_{0}\right\}$ with $x_{0}$ an atom in $X$. Thus, though $\mu\left(\left\{x_{0}\right\}\right)>0$, we have that

$$
\int_{R}\left|f(x)-f\left(x_{0}\right)\right| d \mu(x)=0 .
$$

Hence, it only remains to consider the case in which $R \in \tilde{\mathcal{D}}$. Set $\mathcal{H}_{f}=\{h \in \mathcal{H}$ : $\left.c_{h} \neq 0\right\}$. Then

$$
f=\sum_{h \in \mathcal{H}_{f}} c_{h} h .
$$

Let $\mathcal{J}$ be the function given in (b), Proposition 2.3 For each $h \in \mathcal{H}$, we consider $Q_{h} \in \tilde{\mathcal{D}}$, the dyadic cube given in (h.1), and we separate the function $f$ according to the different levels of resolution. More precisely, we write $f=f_{1}+f_{2}+f_{3}$ with $f_{i}=\sum_{h \in \mathcal{H}_{f_{i}}} c_{h} h, i=1,2,3$, and

$$
\begin{aligned}
& \mathcal{H}_{f_{1}}=\left\{h \in \mathcal{H}_{f}: \mathcal{J}\left(Q_{h}\right)<\mathcal{J}(R)\right\}, \\
& \mathcal{H}_{f_{2}}=\left\{h \in \mathcal{H}_{f}: \mathcal{J}\left(Q_{h}\right) \geq \mathcal{J}(R) \text { and } Q_{h} \cap R \neq \emptyset\right\}, \\
& \mathcal{H}_{f_{3}}=\left\{h \in \mathcal{H}_{f}: \mathcal{J}\left(Q_{h}\right) \geq \mathcal{J}(R) \text { and } Q_{h} \cap R=\emptyset\right\} .
\end{aligned}
$$

It is clear that if $x \in R$ then $f_{3}(x)=0$. Also, since all but a a finite number of terms of the sequence $\mathfrak{C}=\left(c_{h}\right)_{h \in \mathcal{H}}$ are zero, we get that this sequence belongs to the dyadic Carleson class $\mathcal{C}$. So, since $\mathcal{H}$ is an orthonormal basis of $L^{2}(X, \mu)$, from Bessel's inequality we have that

$$
\left\|f_{2}\right\|_{2} \leq\left(\sum_{h \in \mathcal{H}_{f_{2}}}\left|c_{h}\right|^{2}\right)^{1 / 2} \leq C \| \mathfrak{C}_{\mathcal{C}} \mu(R)^{1 / 2}
$$


Hence, by Hölder's inequality and the above estimation of the $L^{2}$-norm of $f_{2}$ we obtain

$$
\begin{aligned}
\int_{R}\left|f(x)-f_{1}\left(x_{0}\right)\right| d \mu(x) & \leq \int_{R}\left|f_{1}(x)-f_{1}\left(x_{0}\right)\right| d \mu(x)+\int_{R}\left|f_{2}(x)\right| d \mu(x) \\
& \leq \int_{R}\left|f_{1}(x)-f_{1}\left(x_{0}\right)\right| d \mu(x)+\left(\int_{R}\left|f_{2}\right|^{2} d \mu\right)^{1 / 2} \mu(R)^{1 / 2} \\
& \leq \int_{R}\left|f_{1}(x)-f_{1}\left(x_{0}\right)\right| d \mu(x)+C\|\mathfrak{C}\|_{\mathcal{C}} \mu(R) .
\end{aligned}
$$

On the other hand, we notice that if $h \in \mathcal{H}_{f_{1}}$ then we have the following two possibilities: $Q_{h} \cap R=\emptyset$ or $R \subset Q_{h}$. In the former case we get that $h(x)=$ $h\left(x_{0}\right)=0$ for each $x \in R$. In the latter, $\mathcal{J}(R)>\mathcal{J}\left(Q_{h}\right)$ and therefore the cube $R$ is a descendant of the cube $Q_{h}$. Hence, from (h.4) we get that $h(x)-h\left(x_{0}\right)=0$ if $x \in R$. So

$$
I_{1}=\int_{R} \sum_{\substack{h \in \mathcal{H}_{f_{1}} \\ Q_{h} \cap R=\emptyset}}\left|c_{h}\right|\left|h(x)-h\left(x_{0}\right)\right| d \mu(x)=0
$$

and

$$
I_{2}=\int_{R} \sum_{\substack{h \in \mathcal{H}_{f_{1}} \\ R \subset Q_{h}}}\left|c_{h}\right|\left|h(x)-h\left(x_{0}\right)\right| d \mu(x)=0 .
$$

Thus

$$
\begin{aligned}
\int_{R}\left|f_{1}(x)-f_{1}\left(x_{0}\right)\right| d \mu(x) & =\int_{R}\left|\sum_{h \in \mathcal{H}_{f_{1}}} c_{h}\left(h(x)-h\left(x_{0}\right)\right) d \mu(x)\right| \\
& \leq I_{1}+I_{2}=0 .
\end{aligned}
$$

\section{Proof of Theorem 1.4}

To prove that the series $\sum_{h \in \mathcal{H}} c_{h} h$ converges, in the sense of the weak-* topology, to a function in $\mathrm{BMO}^{\mathcal{D}}$, we first notice that given an integer $j$ and $x \in X$, from (d.6), (h.1) and (h.2), we get that

$$
\sum_{\substack{h \in \mathcal{H} \\ Q_{h} \in \mathcal{D}^{j}}} c_{h} h(x)
$$

is a finite sum, where $Q_{h}$ is the dyadic cube given in (h.1) for each Haar function $h$. So the function

$$
f^{N}=\sum_{j=-N}^{N} \sum_{\substack{h \in \mathcal{H} \\ Q_{h} \in \mathcal{D}^{j}}} c_{h} h
$$

with $N$ belonging to the positive integers $\mathbb{Z}^{+}$, is well defined. 
Now, we shall prove that $f^{N}$ belongs to $\mathrm{BMO}^{\mathcal{D}}$ for each positive integer $N$. Let $Q_{0}$ be a dyadic cube in $\mathcal{D}$ and let $x_{0} \in Q_{0}$. As in the proof of Theorem 1.3 we only need to consider the case in which $Q_{0} \in \tilde{\mathcal{D}}$. Using the same notation as in the previous section, we consider the set $\mathcal{H}_{f^{N}}=\left\{h \in \mathcal{H}: \mathcal{J}\left(Q_{h}\right)=-N, \ldots, N\right\}$ and rewrite $f^{N}$ as $f^{N}=f_{1}^{N}+f_{2}^{N}+f_{3}^{N}$, with $f_{i}^{N}=\sum_{h \in \mathcal{H}_{f_{i}^{N}}} c_{h} h, i=1,2,3$, and

$$
\begin{aligned}
& \mathcal{H}_{f_{1}^{N}}=\left\{h \in \mathcal{H}_{f^{N}}: \mathcal{J}\left(Q_{h}\right)<\mathcal{J}\left(Q_{0}\right)\right\}, \\
& \mathcal{H}_{f_{2}^{N}}=\left\{h \in \mathcal{H}_{f^{N}}: \mathcal{J}\left(Q_{h}\right) \geq \mathcal{J}\left(Q_{0}\right) \text { and } Q_{h} \cap Q_{0} \neq \emptyset\right\}, \\
& \mathcal{H}_{f_{3}^{N}}=\left\{h \in \mathcal{H}_{f^{N}}: \mathcal{J}\left(Q_{h}\right) \geq \mathcal{J}\left(Q_{0}\right) \text { and } Q_{h} \cap Q_{0}=\emptyset\right\} .
\end{aligned}
$$

Because $f_{3}^{N}(x)=0$ for each $x \in Q_{0}$ we have that

$$
\begin{aligned}
\frac{1}{\mu\left(Q_{0}\right)} \int_{Q_{0}}\left|f^{N}(x)-f_{1}^{N}\left(x_{0}\right)\right| d \mu(x) & =\frac{1}{\mu\left(Q_{0}\right)} \int_{Q_{0}}\left|f_{1}^{N}(x)+f_{2}^{N}(x)-f_{1}^{N}\left(x_{0}\right)\right| d \mu(x) \\
& \leq I_{1}+I_{2},
\end{aligned}
$$

with

$$
I_{1}=\frac{1}{\mu\left(Q_{0}\right)} \int_{Q_{0}}\left|f_{1}^{N}(x)-f_{1}^{N}\left(x_{0}\right)\right| d \mu(x) \quad \text { and } \quad I_{2}=\frac{1}{\mu\left(Q_{0}\right)} \int_{Q_{0}}\left|f_{2}^{N}(x)\right| d \mu(x) .
$$

But, as in the proof of Theorem 1.3 , we have that $f_{1}^{N}(x)-f_{1}^{N}\left(x_{0}\right)=0$ for each $x \in Q_{0}$. Hence $I_{1}=0$. Also, from Hölder's inequality, Bessel's inequality for the orthonormal basis $\mathcal{H}$, and the fact that the sequence $\mathfrak{C}$ belongs to the Carleson class $\mathcal{C}$, we have that

$$
\begin{aligned}
I_{2} & \leq \frac{1}{\mu\left(Q_{0}\right)}\left(\int_{Q_{0}}\left|f_{2}^{N}(x)\right| d \mu(x)\right)^{1 / 2} \mu\left(Q_{0}\right)^{1 / 2} \\
& =\frac{1}{\mu\left(Q_{0}\right)^{1 / 2}}\left\|f_{2}^{N}\right\|_{2} \\
& \leq \frac{1}{\mu\left(Q_{0}\right)^{1 / 2}}\left(\sum_{h \in \mathcal{H}_{f_{2}}}\left|c_{h}\right|^{2}\right)^{1 / 2} \\
& \leq C \| \mathfrak{C}_{\mathcal{C}} \mu\left(Q_{0}\right) .
\end{aligned}
$$

Hence, $f^{N}$ belongs to $\mathrm{BMO}^{\mathcal{D}}$. In the sequel for $N \in \mathbb{Z}^{+}$we shall write, as usual, $\left(u, f^{N}\right)$ to denote the action of the functional $\Phi_{f^{N}}$ over the function $u \in H_{1}^{\mathcal{D}}$, where $\Phi_{f^{N}}$ is the functional given by Proposition 3.8 associated with the function $f^{N}$. That is,

$$
\left(u, f^{N}\right)=\Phi_{f^{N}}(u) .
$$

Now we shall prove that the sequence $\left(f^{N}: N \in \mathbb{Z}^{+}\right)$converges in the sense of the weak-* topology. For this, we prove that it is a Cauchy sequence in this topology. More precisely, we shall see that given $\varepsilon>0$ we have that

$$
\left|\left(u, f^{N}\right)-\left(u, f^{N^{\prime}}\right)\right|<\varepsilon
$$

for $N$ and $N^{\prime}$ large enough and for each function $u \in H_{1}^{\mathcal{D}}$. 
Let $u \in H_{1}^{\mathcal{D}}$. That is,

$$
u=\sum_{n \in \mathbb{Z}^{+}} \lambda_{n} a_{n}, \quad \text { with } \sum_{n \in \mathbb{Z}^{+}}\left|\lambda_{n}\right|<\infty,
$$

where each $a_{n}$ belongs to $\mathcal{A}_{\infty, \mathcal{D}}$ and the convergence of the series is in the $L^{1}(X, \mu)$ norm. For each positive integer $n$ we shall write $Q_{n}$ to denote the dyadic cube that supports the atom $a_{n}$ given in Definition 3.5 with $q=\infty$. Let us take $N^{\prime}<N$. For each couple of integers $\left(J_{1}, J_{2}\right)$ with $J_{1}<J_{2}$ we shall write

$$
f^{J_{1}, J_{2}}=\sum_{j=J_{1}}^{J_{2}} \sum_{\substack{h \in \mathcal{H} \\ Q_{h} \in \mathcal{D}^{j}}} c_{h} h .
$$

So, $f^{N}-f^{N^{\prime}}=f^{N^{\prime}, N}+f^{-N,-N^{\prime}}$ and then from Proposition 3.8 we can pass to integral form in the following way:

$$
\begin{aligned}
\left|\left(u, f^{N}\right)-\left(u, f^{N^{\prime}}\right)\right| & =\left|\sum_{n \in \mathbb{Z}^{+}} \lambda_{n}\left(a_{n}, f^{N^{\prime}, N}+f^{-N,-N^{\prime}}\right)\right| \\
& \leq \sum_{n \in \mathbb{Z}^{+}}\left|\lambda_{n}\right|\left|\int_{Q_{n}} a_{n}(x)\left(f^{N^{\prime}, N}(x)+f^{-N,-N^{\prime}}(x)\right) d \mu(x)\right| \\
& \leq I_{1}+I_{2},
\end{aligned}
$$

where

$$
I_{1}=\sum_{n \in \mathbb{Z}^{+}}\left|\lambda_{n}\right|\left|\int_{Q_{n}} a_{n}(x) \sum_{j=-N}^{-N^{\prime}} \sum_{\substack{h \in \mathcal{H} \\ Q_{h} \in \mathcal{D}^{j}}} c_{h} h(x) d \mu(x)\right|
$$

and

$$
I_{2}=\sum_{n \in \mathbb{Z}^{+}}\left|\lambda_{n}\right|\left|\int_{Q_{n}} a_{n}(x) \sum_{j=N^{\prime}}^{N} \sum_{\substack{h \in \mathcal{H} \\ Q_{h} \in \mathcal{D}^{j}}} c_{h} h(x) d \mu(x)\right| .
$$

Since the same argument is used to prove that both $I_{1}$ and $I_{2}$ are less than $\varepsilon$, we shall only present with some detail the case $I_{2}$. Notice that for each positive integer $n$ we get that

$$
\int_{Q_{n}} a_{n}(x) f^{N^{\prime}, N}(x) d \mu(x)=\int_{Q_{n}} a_{n}(x) \sum_{i=1}^{3} f_{n, i}(x) d \mu(x),
$$

where $f_{n, i}(x)=\sum_{h \in \mathcal{H}_{n, i}} c_{h} h(x)$ and

$$
\begin{aligned}
& \mathcal{H}_{n, 1}=\left\{h \in \mathcal{H}_{f^{N^{\prime}, N}}: \mathcal{J}\left(Q_{h}\right)<\mathcal{J}\left(Q_{n}\right)\right\}, \\
& \mathcal{H}_{n, 2}=\left\{h \in \mathcal{H}_{f^{N^{\prime}, N}}: \mathcal{J}\left(Q_{h}\right) \geq \mathcal{J}\left(Q_{n}\right) \text { and } Q_{h} \cap Q_{n} \neq \emptyset\right\}, \\
& \mathcal{H}_{n, 3}=\left\{h \in \mathcal{H}_{f^{N^{\prime}, N}}: \mathcal{J}\left(Q_{h}\right) \geq \mathcal{J}\left(Q_{n}\right) \text { and } Q_{h} \cap Q_{n}=\emptyset\right\},
\end{aligned}
$$

with

$$
\mathcal{H}_{f^{N^{\prime}, N}}=\left\{h \in \mathcal{H}: \mathcal{J}\left(Q_{h}\right)=N^{\prime}, \ldots, N\right\}
$$


Thus, for each positive integer $n$ we have that $f_{n, 3}(x)=0$ if $x \in Q_{n}$, and we obtain

$$
I_{2} \leq \sum_{n \in \mathbb{Z}^{+}}\left|\lambda_{n}\right|\left|A_{n}+B_{n}\right|
$$

where

$$
A_{n}=\int_{Q_{n}} a_{n}(x) f_{n, 1}(x) d \mu(x)
$$

and

$$
B_{n}=\int_{Q_{n}} a_{n}(x) f_{n, 2}(x) d \mu(x) .
$$

But, since $a$ is a dyadic $\infty$-atom and $f_{n, 1}(x)-f_{n, 1}\left(x_{n}\right)=0$ if $x, x_{n}$ belong to $Q_{n}$, we have that

$$
A_{n}=\int_{Q_{n}} a_{n}(x)\left(f_{n, 1}(x)-f_{n, 1}\left(x_{n}\right)\right) d \mu(x)=0 .
$$

Hence we only need to show that $\sum_{n \in \mathbb{Z}^{+}}\left|\lambda_{n}\right|\left|B_{n}\right|<\varepsilon$ for $N^{\prime}$ large enough. Notice that for $K \in \mathbb{Z}^{+}$, from Hölder's inequality and from Bessel's inequality we get that

$$
\begin{aligned}
\sum_{n \in \mathbb{Z}^{+}}\left|\lambda_{n}\right|\left|B_{n}\right| \leq & \sum_{n \in \mathbb{Z}^{+}}\left|\lambda_{n}\right|\left\|a_{n}\right\|_{L^{2}\left(Q_{n}\right)}\left\|f_{n, 2}\right\|_{L^{2}\left(Q_{n}\right)} \\
= & \sum_{n \in \mathbb{Z}^{+}}\left|\lambda_{n}\right|\left\|a_{n}\right\|_{L^{2}\left(Q_{n}\right)}\left(\sum_{\mathcal{H}_{n, 2}}\left|c_{h}\right|^{2}\right)^{1 / 2} \\
\leq & \sum_{n=1}^{K}\left|\lambda_{n}\right|\left\|a_{n}\right\|_{L^{2}\left(Q_{n}\right)}\left(\sum_{\mathcal{H}_{n, 2}}\left|c_{h}\right|^{2}\right)^{1 / 2} \\
& +\sum_{n=K+1}^{\infty}\left|\lambda_{n}\right|\left\|a_{n}\right\|_{L^{2}\left(Q_{n}\right)}\left(\sum_{\mathcal{H}_{n, 2}}\left|c_{h}\right|^{2}\right)^{1 / 2} .
\end{aligned}
$$

But as the sequence $\left(c_{h}: h \in \mathcal{H}\right)$ belongs to the Carleson class we have that

$$
\left(\sum_{\mathcal{H}_{n, 2}}\left|c_{h}\right|^{2}\right)^{1 / 2}<C \mu\left(Q_{n}\right)^{1 / 2}
$$

and hence

$$
\left(\sum_{\mathcal{H}_{n, 2}}\left|c_{h}\right|^{2}\right)^{1 / 2}<\varepsilon
$$


for $N^{\prime}$ large enough. So, from (5.1) and the fact that $a$ is a dyadic $\infty$-atom we get that

$$
\begin{aligned}
\sum_{n=K+1}^{\infty}\left|\lambda_{n}\right|\left\|a_{n}\right\|_{L^{2}\left(Q_{n}\right)}\left(\sum_{\mathcal{H}_{n, 2}}\left|c_{h}\right|^{2}\right)^{1 / 2} & \leq \sum_{n=K+1}^{\infty}\left|\lambda_{n}\right|\left\|a_{n}\right\|_{L^{2}\left(Q_{n}\right)}\left(\mu\left(Q_{n}\right)\right)^{1 / 2} \\
& \leq \sum_{n=K+1}^{\infty}\left|\lambda_{n}\right|\left\|a_{n}\right\|_{\infty} \mu\left(Q_{n}\right) \\
& \leq \sum_{n=K+1}^{\infty}\left|\lambda_{n}\right| \\
& \leq \varepsilon
\end{aligned}
$$

for $K$ large enough since the series $\sum_{n=1}^{\infty}\left|\lambda_{n}\right|$ converges. On the other hand, from (5.2) we get

$$
\sum_{n=1}^{K}\left|\lambda_{n}\right|\left\|a_{n}\right\|_{L^{2}\left(Q_{n}\right)}\left(\sum_{\mathcal{H}_{n, 2}}\left|c_{h}\right|^{2}\right)^{1 / 2}<\varepsilon \sum_{n=1}^{K}\left|\lambda_{n}\right|\left\|a_{n}\right\|_{L^{2}\left(Q_{n}\right)} .
$$

Hence, the sequence $\left(f^{N}: N \in \mathbb{Z}^{+}\right)$is a Cauchy sequence in the weak-* topology.

\section{REFERENCES}

[1] H. Aimar, Construction of Haar type bases on quasi-metric spaces with finite Assouad dimension, Anal. Acad. Nac. Cienc. Exactas Fís. Nat. (Buenos Aires) 54 (2002), 67-82.

[2] H. Aimar and A. Bernardis, Wavelet characterization of functions with conditions on the mean oscillation, Wavelet theory and harmonic analysis in applied sciences (Buenos Aires, 1995), 15-32, Appl. Numer. Harmon. Anal., Birkäuser Boston, 1997. MR 1463237.

[3] H. Aimar, A. Bernardis and B. Iaffei, Multiresolution approximations and unconditional bases on weighted Lebesgue spaces on spaces of homogeneous type, J. Approx. Theory 148 (2007) 12-34. MR 2356573

[4] H. Aimar, A. Bernardis and B. Iaffei, Comparison of Hardy-Littlewood and dyadic maximal functions on spaces of homogeneous type, J. Math. Anal. Appl. 312 (2005) 105-120. MR 2175208

[5] H. Aimar, A. Bernardis and L. Nowak, On Haar bases for generalized dyadic Hardy spaces, Rocky Mountain J. Math. 43 (2013), 697-712. MR 3093261

[6] H. Aimar, A. Bernardis and L. Nowak, Equivalence of Haar bases associated with different dyadic systems, J. Geom. Anal. 21 (2011), 288-304. MR 2772074

[7] L. Carleson, An explicit unconditional basis in $H^{1}$, Bull. Sci. Math. (2) 104 (1980), 405-416. MR 0602408

[8] S.-Y. A. Chang and R. Fefferman, Some recent developments in Fourier analysis and $H^{p}$ theory on product domains, Bull. Amer. Math. Soc. (N.S.) 12 (1985), 1-43. MR 0766959

[9] M. Christ, $A T(b)$ theorem with remarks on analytic capacity and the Cauchy integral, Colloq. Math. 60/61 (1990), 601-628. MR 1096400.

[10] R. Coifman and G. Weiss, Extensions of Hardy spaces and their use in analysis, Bull. Amer. Math. Soc. 83 (1977), 569-645. MR 0447954.

[11] J. Conway, A course in functional analysis, Second edition. Graduate Texts in Mathematics, 96, Springer, 1990. MR 1070713

[12] I. Daubechies, Ten lectures on wavelets, CBMS-NSF Regional Conference Series in Applied Mathematics, 61, SIAM, 1992. MR 1162107 
[13] E. Hernández and G. Weiss, A first course on wavelets, Studies in Advanced Mathematics, CRC Press, 1996. MR 1408902

[14] E. Harboure, O. Salinas and B. Viviani, Wavelet expansions for $\operatorname{BMO}_{\rho}(w)$-functions. Math. Nachr. 281 (2008), 1747-1763. MR 2473326

[15] P. Lemarié and Y. Meyer, Ondelettes et bases hilbertiennes, Rev. Mat. Iberoamericana 2 (1986), 1-18. MR 0864650

[16] R. Macías and C. Segovia, Lipschitz functions on spaces of homogeneous type, Adv. in Math. 33 (1979), 257-270. MR 0546295.

[17] Y. Meyer, Wavelets and operators, Cambridge Studies in Advanced Mathematics, 37, Cambridge University Press, 1992. MR 1228209

[18] P. Wojtaszczyk, A mathematical introduction to wavelets, London Mathematical Society Student Texts, 37, Cambridge University Press, 1997. MR 1436437.

R. Crescimbeni

Departamento de Matemática (FaEA-UNCOMA), IITCI (CONICET-UNCOMA), Neuquén, Argentina

raquel.crescimbeni@faea.uncoma.edu.ar

L. Nowak ${ }^{凶}$

Departamento de Matemática (FaEA-UNCOMA), IITCI (CONICET-UNCOMA), Neuquén, Argentina

luis.nowak@faea. uncoma.edu.ar

Received: July 26, 2016

Accepted: June 6, 2017 